\title{
MOOD STABILIZER THERAPY AND PRAVASTATIN: HIGHER RISK FOR ADVERSE SKIN REACTIONS?
}

\author{
Alice Walder ${ }^{1}$, Pierre Baumann ${ }^{2}$ \\ Psychiatric Hospital Sanatorium Kilchberg, Alte Landstrasse 70-84, CH-8802 Kilchberg, Switzerland ${ }^{1}$; Department of \\ Psychiatry, Vaudois University Hospital Centre (DP-CHUV), Site de Cery, CH-1008 Prilly-Lausanne, Switzerland ${ }^{2}$
}

\begin{abstract}
Summary: We report on a serious side effect in a severely depressed 55-year-old woman, who presented an erythematous pigmented skin rash on the whole body under combination treatment with antidepressants, atypical antipsychotic drugs, the mood stabilizer lithium and the lipid-lowering drug pravastatin. The skin rash effect was most probably due, in first line, to olanzapine, but the cutaneous skin condition was triggered and aggravated by pravastatin, a 3-hydoxy-3-methylglutaryl-coenzyme A-(HMG-CoA)-reductase inhibitor, and lithium medication. The allergic reaction started to develop after co-administration of pravastatin. Therefore, the combination of atypical antipsychotics with statins should be carefully monitored and the benefits and disadvantages should be balanced.
\end{abstract}

Key words: Adverse skin reaction; Mood stabilizer and lipid-lowering drugs; Depression; Drug monitoring

\section{Introduction}

Various pharmacological strategies were proposed during the last decade to treat severe unipolar depression, among which augmentation therapies are the most common. The use of selective serotonin reuptake inhibitors (SSRI), combined with one or more mood stabilizers is well established (10). Augmentation therapies can cause several side effects and therefore benefits and risks have to be carefully balanced (12). Many drugs including SSRI, lithium, anticonvulsants or antipsychotics can reportedly cause allergic side reactions, including skin lesions $(1,5)$. Mood stabilizers present the highest risk for severe adverse skin reactions and the combination of such agents may considerably increase the incidence of adverse side effects (11). If these drugs are coadministered with a lipid-lowering statin therapy, which itself can cause allergic side effects $(6,8)$, these patients should be carefully controlled to minimize the risk to occur a life-threatening situation. We report on a depressed patient, who presented an erythematous pigmented skin rash while treated with antidepressants, mood stabilizers and the lipid-lowering drug pravastatin. This situation was observed in the context of an international pharmacovigilance program in psychiatry (AMSP). It aims at registering and evaluating severe adverse drug reactions in a continuous open-end study under the conditions of routine clinical treatment (3)

\section{Case report}

The patient (female, 55 years old) was admitted to our hospital as she suffered from a severe depressive episode with psychotic auditory hallucinations, accompanied by numerous attempted suicides. The patient experienced her first depressive episode when she was 17 years old. During the preceding years, the depression aggravated and she displayed simultaneously agoraphobia and panic attacks. The frequently occurring depressive episodes were treated with several antidepressant drugs, but medication was often discontinued because of ineffectiveness or her predisposition to present adverse effects. Treatments with SSRI as paroxetine, or with mirtazapine and venlafaxine were often discontinued because of gastrointestinal side effects or insufficient therapeutic response. Because all other classical treatments failed, tricyclic antidepressants such as imipramine, trimipramine and maprotiline were prescribed during different depressive episodes in the preceding years. The patient never improved, but she displayed a higher than $10 \%$ increase in weight within one year. Because of the severity of her depression with the presence of continuous suicidal ideation, the patient who also presented a personality disorder was unable to participate in an education group for lifestyle or perform more physical activity. Mood stabilizers such as carbamazepine, olanzapine and valproate caused mild eczematous skin lesions or strong sedation. The patient never 
suffered from skin problems or any allergic diathesis in childhood.

Before admission (on day 0 ), the patient was polymedicated with $100 \mathrm{mg} /$ day sertraline, $625 \mathrm{mg} /$ day lithium carbonate, $60 \mathrm{mg} /$ day aripiprazole, $200 \mathrm{mg}$ /day quetiapine, 4 $\mathrm{mg} /$ day lorazepam, $7.5 \mathrm{mg} /$ day magnesium and $0.05 \mathrm{mg} /$ day levothyroxine. After admission, the dose of the antidepressant medication sertraline was increased from $100 \mathrm{mg} /$ day to $150 \mathrm{mg} /$ day after 2 days and to $200 \mathrm{mg} /$ day after another 8 days. Because of tiredness during the day, lorazepam medication was reduced after 18 days to $3 \mathrm{mg} /$ day and after 22 days to $2 \mathrm{mg} /$ day after admission. Despite the high dose of aripiprazole (60 mg/day) the psychotic symptoms remained during hospitalisation. Therefore, treatment with this drug was stopped after 41 days, by a stepwise reduction of its dose $(45 \mathrm{mg} /$ day after 33 days; $30 \mathrm{mg} /$ day after another 3

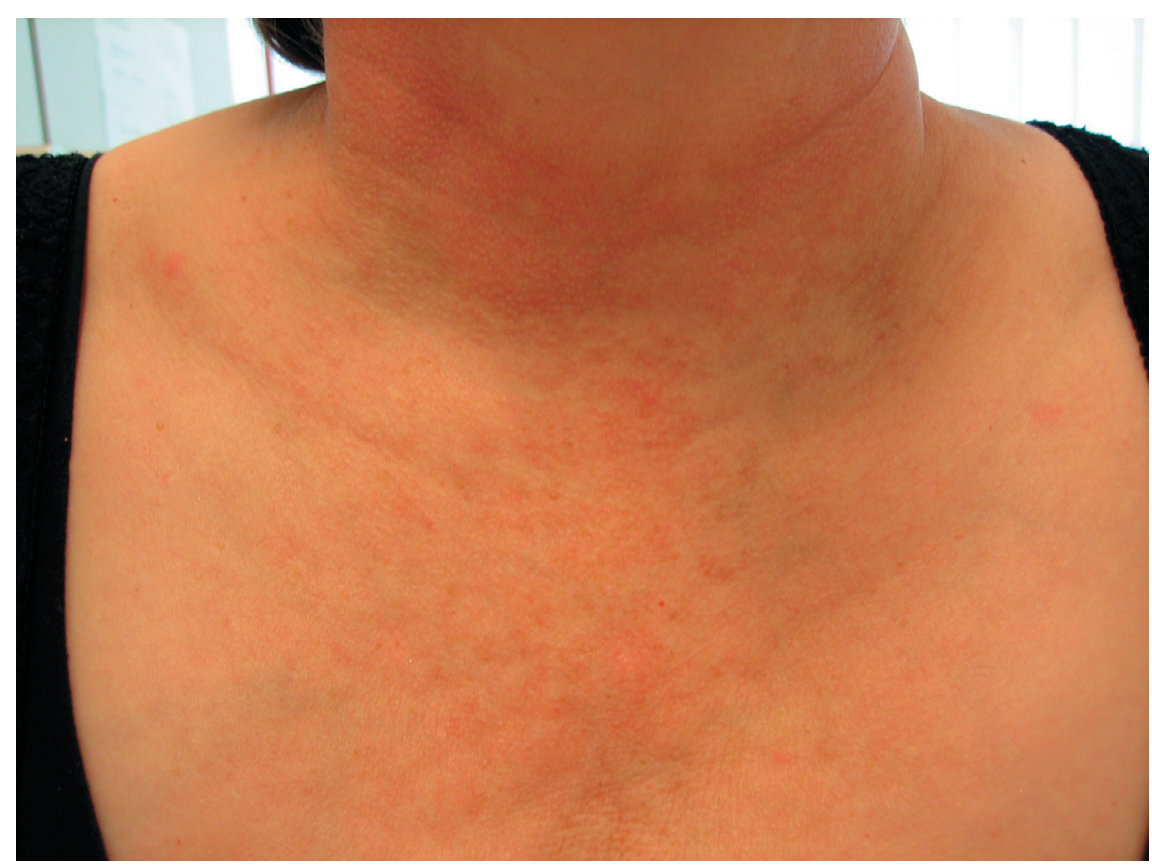

Fig. 1: Pigmented skin rash on the neck and necklin.

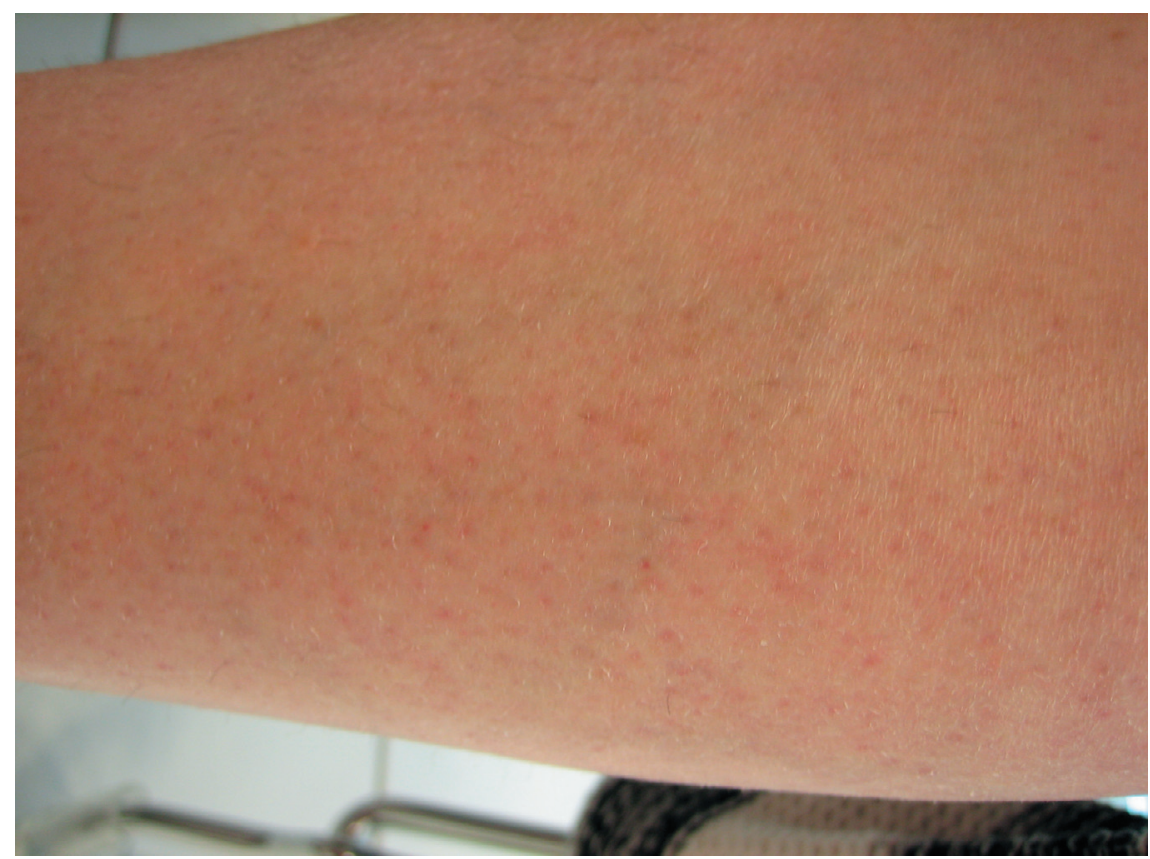

Fig. 2: Pigmented skin rash on the shanks. 
days, and then its dose was halved every 2 till 3 days). On day 26, lithium carbonate was increased to $900 \mathrm{mg} /$ day and on day 28 , a treatment with $10 \mathrm{mg} /$ day olanzapine was commenced. Earlier experience had shown, that this medication was of a high antipsychotic benefit to this the patient, but it resulted also in adverse side reactions as massive weight gain and hyperlipidemia. As there was a known family risk for them, a statin therapy was initiated on day 29 . The psychotic symptoms decreased rapidly, but a skin lesion was observed on day 32 . On day 37 , the patient presented with a diffuse pruritic, pigmented skin rash on the whole body, especially on her neck, neckline and shanks (Fig. 1 and 2). On this day, the plasma concentration of sertraline $(0.15 \mu \mathrm{mol} / 1)$ was within the recommended range (0.03-0.16 $\mu \mathrm{mol} / 1)$, while that of olanzapine $(50 \mathrm{nmol} / \mathrm{l})$ was below it $(64-256 \mathrm{nmol} / \mathrm{l})$. The fact that despite the high dose of aripiprazole ( $45 \mathrm{mg} /$ day on day 37 ), its plasma concentration was close to the limit of quantitation $(0.02$ $\mu \mathrm{mol} / 1$ ) (recommended range: $0.2-0.67 \mu \mathrm{mol} / 1$ ) suggests poor compliance and may explain its lack of efficacy. Pravastatin was administered during 9 days, but due its possible implication in the occurrence of these adverse effects, its prescription was discontinued on day 38. Laboratory findings showed a temporary serologic eosinophilia $(7.1 \%$ and $9.6 \%$, days 38 and 40, respectively) (normal range: 0-6\%). The other biochemical parameters were normal, especially absence of leucocytosis $\left(7.1 \times 10^{3} / \mu 1\right.$ ) (normal range: $\left.4-10.0 \times 10^{3} / \mu 1\right)$, no increase of C-reactive protein ( $7 \mathrm{mg} / \mathrm{L})$ (normal range: $<8 \mathrm{mg} / \mathrm{L}$ ) or erythrocyte sedimentation rate $(11 \mathrm{~mm})$ (normal range: $3-11 \mathrm{~mm}$ ).

Despite local therapy with the corticosteroid betamethasone, the skin rash kept the same intensity; additionally, mild pruritic symptoms appeared in the eyes of the patient. As a few years ago she already had a mild eczematous skin lesion episode while treated with olanzapine, this medication was nevertheless administered for an additional time period of 15 days (i.e. totally 21 days), with frequent laboratory controls, because the suicidal ideas and psychotic symptoms slowly disappeared. Unfortunately, the allergic symptoms remained and medication with olanzapine had to be stopped on day 49 of hospitalisation and replaced by $1 \mathrm{mg} /$ day risperidone on the following day. Subsequently the pruritic symptoms declined and the serologic laboratory findings normalised. In particular, the skin rash symptoms decreased slowly during the following 18 days after discontinuation of olanzapine medication till she left the hospital but on the other hand, depressive symptoms increased. Finally, this severe depressive episode required an electroconvulsive therapy, which the patient had refused till this term.

\section{Discussion}

This patient suffered from therapy-resistant and psychotic depression, pronounced suicidality as well as from a personality disorder, and presented in some stades of her illness a questionable compliance. Polymedication was indicated at her hospitalisation, comprising among other drugs a combination of an antidepressant with an antipsychotic agent, in accordance with some recent guideline (9).

Olanzapine alone might be sufficient to provoke a rash $(2,4)$ since there is earlier evidence of skin problems with this drug in this patient. Moreover, olanzapine may lead to hyperlipidemia and other metabolic problems (7). Therefore, especially in patients with cardiovascular risk factors, an additional lipid-lowering therapy is recommended, which itself can cause allergic side effects. It is well-established that statins like pravastatin modify the composition of skin

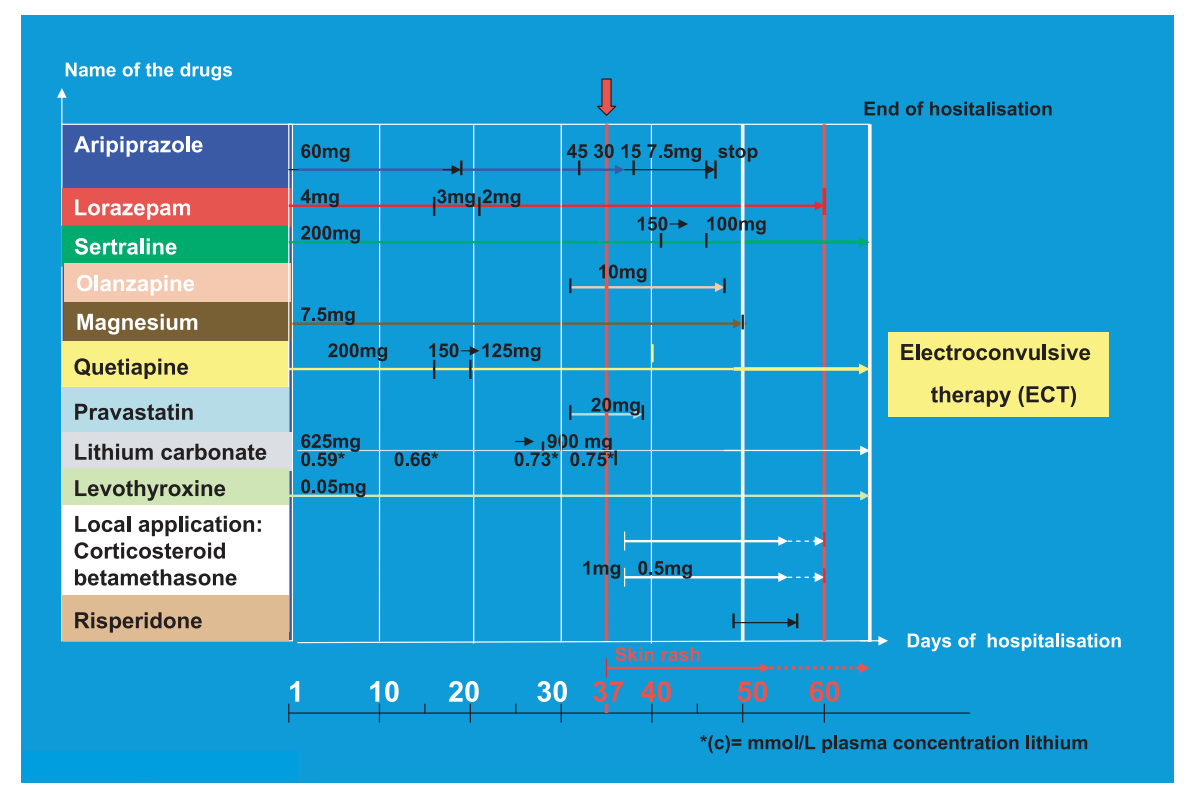

Graph 1: Appearance of the pigmented skin rash. 
keratin and this results in a higher vulnerability of the skin to other drugs $(6,8)$. As in our patient the adverse skin reaction started to develop after co-administration of pravastatin, it was probably not primarily due to olanzapine, but modifications of the skin condition by pravastatin and lithium (1) increased the risk for olanzapine to provoke it. Polymedication with aripiprazole, sertraline and quetiapine was initiated several months before hospitalisation. In the course of hospitalization the complex medication regimen was reduced and simplified. Some of the medications were discontinued. Therefore it seems improbable that these drugs significantly contributed to the adverse effect. The generalized skin reaction seems to be allergic and the temporary serologic eosinophilia developed to normal, when the pravastatin medication was stopped. Allergological skin tests as Prick-tests, Scratch-tests and Epicutaneous-tests were proposed to the patient, but reexposure to olanzapine was not performed, because she feared reappearance of a severe adverse reaction and in this critical situation of permanent suicidal ideas of the patient, these skin tests were abandoned.

In conclusion, comedication of antidepressants or antipsychotics with the lipid-lowering statin therapy should be carefully kept under surveillance and the potentiating effect of the latter for skin adverse side reactions should be recognised.

\section{References}

1. Chan HH, Wing Y, Su R, Van Krevel, Lee S. A control study of the cutaneous side effects of chronic lithium therapy. J Affect Disord. 2000;57(1-3):107-13.

2. Christen S, Gueissaz F, Anex R, Zullino DF. Acute generalized exanthematous pustulosis induced by olanzapine. Acta Medica (Hradec Kralove) 2006;49(1): 75-6.

3. Degner D, Grohmann R, Kropp S, et al. Severe adverse drug reactions of antidepressants: results of the German multicenter drug surveillance program AMSP. Pharmacopsychiatry. 2004;37 Suppl 1:39-45.

4. Duggal MK, Singh A, Arunabh, Lolis JD, Guzik HJ. Olanzapine-induced vasculitis. Am J Geriatr Pharmacother. 2005;3(1):21-4.

5. Jhirwal OP, Parsad D, Basu D. Skin hyperpigmentation induced by olanzapine, a novel antipsychotic agent. Int J Dermatol. 2004;43(10):778-9.

6. Krasovec M, Elsner P, Burg G. Generalized eczematous skin rash possibly due to HMG-CoA reductase inhibitors. Dermatology. 1993;186(4):248-52.

7. Newcomer JW, Haupt DW. The metabolic effects of antipsychotic medications. Can J Psychiatry. 2006;51(8):480-51.

8. Proksch E. Antilipemic drug-induced skin manifestations. Hautarzt 1995;46(2) 76-80.

9. Sartorius N, Baghai TC, Baldwin DS, et al. Antidepressant medications and other treatments of depressive disorders: a CINP Task Force report based on a review of evidence. Int J Neuropsychopharmacol. 2007;10 Suppl 1:1-207.

10. Souery D, Papakostas GI, Trivedi MH. Treatment-resistant depression. J Clin Psychiatry. 2006;67 Suppl 6:16-22.

11. Warnock JK, Morris DW. Adverse cutaneous reactions to mood stabilizers. Am J Clin Dermatol. 2003;4(1):21-30.

12. Newcomer JW, Haupt DW. The metabolic effects of antipsychotic medications. Can J Psychiatry. 2006;51(8):480-51.

\section{Corresponding author:}

Dr. Alice Walder, Head of Internal Medicine, Psychiatric Hospital Sanatorium Kilchberg, Alte Landstrasse 70-84, CH-8802 Kilchberg, Switzerland; e-mail: a.walder@sanatorium-kilchberg.ch 